\title{
Azimuthally invariant laser polarimetry of polycrystalline smears of biological liquids
}

\author{
Yu.O. Ushenko' , O.V. Dubolazov ${ }^{1}$, A.V. Motrich ${ }^{1}$, G.B. Bodnar ${ }^{2}$ \\ ${ }^{I}$ Chernivtsi National University, Optics and Publishing Department \\ 2, Kotsyubinsky str., 58012 Chernivtsi, Ukraine \\ ${ }^{2}$ Bukovinian State Medical University, 58000 Chernivtsi, Ukraine \\ E-mail: a.dubolazov@chnu.edu.ua
}

\begin{abstract}
The model based on Mueller-matrix description of mechanisms providing optical anisotropy typical for polycrystalline smears of liquor (including optical activity, birefringence as well as linear and circular dichroism) has been suggested. Within the statistical analysis of such distributions, the objective criteria of differentiation of smears of liquor taken from dead patient at different moments after his death have been determined. From the viewpoint of probative medicine, the operational characteristics (sensitivity, specificity and accuracy) of the method of Mueller-matrix reconstruction of optical anisotropy parameters have been found and the efficiency of this method in another task - diagnostics of diseases of internal organs of rats - has been demonstrated.
\end{abstract}

Keywords: polarimetry, liquor, laser image, biological fluids.

Manuscript received 12.11.14; revised version received 27.02.15; accepted for publication 27.05.15; published online 08.06.15.

\section{Introduction}

Biological tissues and fluids represent structurally inhomogeneous media with absorption. To describe interactions of polarized light with these complex systems, the most general approaches based on Muellermatrix formalism are required. Nowadays in biological and medical investigations, many practical techniques based on measurement and analysis of Mueller matrices of investigated samples are used [1-12]. In recent 10-15 years, a separate approach - laser polarimetry [13] - was formed in matrix optics. On its basis, the interactions between the set of statistical moments of the $1^{\text {st }}-4^{\text {th }}$ orders characterizing the Mueller-matrix elements distribution and parameters of linear birefringence of fibrillar protein networks of human biological tissues have been determined. It enabled to diagnose oncological changes of skin derma, epithelial and connective tissue of women's reproductive sphere organs etc. [18-21]. In addition, laser polarimetry techniques require further development and generalization.

Firstly, not all elements of Mueller matrix prove to be convenient for characterizing biological samples. The reason of this is the azimuthal dependence of the majority of matrix elements - generally 12 of 16 elements change at rotation of the sample around the probing axis.

Secondly, the spectrum of mechanisms of optical anisotropy of biological layers is not confined to linear birefringence only. Taking into consideration the impact of other mechanisms, circular birefringence as well as linear and circular dichroism are topical in the aspect of enlarging the range of diagnostic techniques. 
Thirdly, there is a wide range of optically anisotropic biological objects, for which laser polarimetry techniques did not spread widely. Biological fluids - blood and its plasma, urine, bile, saliva and others - belong to them. The objects of this class are easily accessible and do not require the traumatic surgery of biopsy.

This research is focused on generalization of optical anisotropy of optically thin layers of liquor films and histological sections of the internal organs of healthy and diabetic rats and the development of the method of "azimuthally stable" Mueller-matrix reconstruction of anisotropy parameters of polycrystalline networks.

\section{Brief theoretical background}

Our work is based on modeling representations of phase anisotropy (optical activity and linear birefringence) of the polycrystalline structure of the films of blood plasma. In this approximation, the experimentally measured matrices have the following symmetry [22-27]:

$$
\{F\}=\{\Omega\}\{D\}=f_{11}^{-1} \times\left\|\begin{array}{cccc}
1 & 0 & 0 & 0 \\
0 & f_{22} & f_{23} & f_{24} \\
0 & f_{32} & f_{33} & f_{34} \\
0 & f_{42} & f_{43} & f_{44}
\end{array}\right\| .
$$

Here, $\{\Omega\}$ is the Mueller matrix circular birefringence or optical activity of amino acid molecules

$$
\begin{aligned}
& \{\Omega\}=\left\|\begin{array}{cccc}
1 & 0 & 0 & 0 \\
0 & \omega_{22} & \omega_{23} & 0 \\
0 & \omega_{32} & \omega_{33} & 0 \\
0 & 0 & 0 & 1
\end{array}\right\|, \\
& \omega_{i k}=\left\{\begin{array}{l}
\omega_{22}=\omega_{33}=\cos 2 \theta ; \\
\omega_{23}=-\omega_{32}=\sin 2 \theta .
\end{array}\right.
\end{aligned}
$$

where $\theta$ is the rotation angle of polarization plane of the light beam transformed by amino acids.

Linear birefringence of amino acids polypeptide chains characterized by Mueller matrix $\{D\}$ is of the following form:

$$
\begin{aligned}
& \{D\}=\left\|\begin{array}{llll}
1 & 0 & 0 & 0 \\
0 & d_{22} & d_{23} & d_{24} \\
0 & d_{32} & d_{33} & d_{34} \\
0 & d_{42} & d_{43} & d_{44}
\end{array}\right\|, \\
& d_{i k}=\left\{\begin{array}{l}
d_{22}=\cos ^{2} 2 \rho+\sin ^{2} 2 \rho \cos \delta, \\
d_{23}=d_{32}=\cos 2 \rho \sin 2 \rho(1-\cos \delta), \\
d_{33}=\sin ^{2} 2 \rho+\cos ^{2} 2 \rho \cos \delta, \\
d_{42}=-d_{24}=\sin 2 \rho \sin \delta, \\
d_{34}=-d_{43}=\cos 2 \rho \sin \delta, \\
d_{44}=\cos \delta .
\end{array}\right.
\end{aligned}
$$

Here, $\rho$ is the direction of optical axes; $\delta=\frac{2 \pi}{\lambda} \Delta n l-$ phase shift between linearly polarized orthogonal components of light beam amplitude; $\lambda$ - wavelength; $\Delta n$ - birefringence; $l$ - geometrical thickness.

$$
\{M\}=\prod_{i=1}^{4}\left\{M_{i}\right\}=M_{11}^{-1} \times\left\|\begin{array}{cccc}
1 & M_{12} & M_{13} & M_{14} \\
M_{21} & M_{22} & M_{23} & M_{24} \\
M_{31} & M_{32} & M_{33} & M_{34} \\
M_{41} & M_{42} & M_{43} & M_{44}
\end{array}\right\| \text {. (4) }
$$

Here, $\left\{M_{i}\right\}$ is the Mueller matrix that characterizes the phase anisotropy $(\{\Omega\},\{D\})$ and also amplitude anisotropy ( $\{\Phi\},\{\Psi\})$ of amino acid molecules and their aggregates.

\section{Circular dichroism}

$\{\Phi\}=\left\|\begin{array}{cccc}1 & 0 & 0 & \phi_{14} \\ 0 & \phi_{22} & 0 & 0 \\ 0 & 0 & \phi_{33} & 0 \\ \phi_{41} & 0 & 0 & 1\end{array}\right\|$,
$\phi_{i k}=\left\{\begin{array}{l}\phi_{22}=\phi_{33}=\frac{1-C^{2}}{1+C^{2}} \\ \phi_{14}=\phi_{41}= \pm \frac{2 C}{1+C^{2}} .\end{array}\right.$

Here, $C=\frac{g_{\otimes}-g_{\oplus}}{g_{\otimes}+g_{\oplus}}, g_{\otimes}, \quad g_{\oplus}$ are the absorption indices of left- $(\otimes)$ and right-hand $(\oplus)$ circularly polarized components of light beam amplitude.

Linear dichroism

$$
\begin{aligned}
& \{\Psi\}=\left\|\begin{array}{cccc}
1 & \varphi_{12} & \varphi_{13} & 0 \\
\varphi_{21} & \varphi_{22} & \varphi_{23} & 0 \\
\varphi_{31} & \varphi_{32} & \varphi_{33} & 0 \\
0 & 0 & 0 & \varphi_{44}
\end{array}\right\|, \\
& \varphi_{i k}=\left\{\begin{array}{l}
\varphi_{12}=\varphi_{21}=(1-\Delta \tau) \cos 2 \rho, \\
\varphi_{13}=\varphi_{31}=(1-\Delta \tau) \sin 2 \rho, \\
\varphi_{22}=(1+\Delta \tau) \cos ^{2} 2 \rho+2 \sqrt{\Delta \tau} \sin ^{2} 2 \rho, \\
\varphi_{23}=\varphi_{32}=(1-\Delta \tau) \sin 2 \rho, \\
\varphi_{33}=(1+\Delta \tau) \sin ^{2} 2 \rho+2 \sqrt{\Delta \tau} \cos ^{2} 2 \rho, \\
\varphi_{44}=2 \sqrt{\Delta \tau} .
\end{array}\right.
\end{aligned}
$$

Here, $\Delta \tau=\frac{\tau_{x}}{\tau_{y}}, \quad\left\{\begin{array}{l}\tau_{x}=\tau \cos \rho \\ \tau_{y}=\tau \sin \rho\end{array}, \quad \tau_{x}, \quad \tau_{y} \quad\right.$ are the absorption coefficients of linearly polarized orthogonal components of light beam amplitude.

$$
\{M\}=\{\Phi\}\{\Psi\}\{D\}\{\Omega\} \text {. }
$$

For analytical and practical application (7), we used the data of investigations $[1,2,4]$. Here, it is shown that 
the following elements of matrix $\{M\}$ as well as their combinations are azimuthally stable, independent of the sample rotation angle $\Theta$

$$
\begin{aligned}
& \left\{\begin{array}{l}
M_{11}(\Theta)=\text { const } ; M_{14}(\Theta)=\text { const, } \\
M_{41}(\Theta)=\text { const } ; M_{44}(\Theta)=\text { const, }
\end{array}\right. \\
& \left\{\begin{array}{l}
{\left[M_{22}+M_{33}\right](\Theta) \equiv \Sigma M_{22 ; 33}(\Theta)=\text { const, }} \\
{\left[M_{23}-M_{32}\right](\Theta) \equiv \Delta M_{23 ; 32}(\Theta)=\text { const. }}
\end{array}\right.
\end{aligned}
$$

\section{Analysis and discussion of experimental data}

The measurements of coordinate distributions of Mueller-matrix elements (distribution of values in the film plane of blood plasma) were performed in the setup (Fig. 1) of the standard Stokes-polarimeter [13].

Illumination of the sample under study was performed by the parallel $\left(\varnothing=10^{4} \mu \mathrm{m}\right)$ laser beam of He-Ne $(\lambda=0.6328 \mu \mathrm{m}, W=5.0 \mathrm{~mW})$. The polarization light source consisted of quarter-wave plates 3,5 and polarizer 4 , it formed a right circularly polarized beam. Blood plasma films were placed in the focal plane of polarization microobjective 7 (focal distance $30 \mathrm{~mm}$, aperture 0.1 , magnification $4 \times$ ). Behind the (Fourier) focal plane the vignetting diaphragm was located, its size changed within the range from 2 up to 300 pix. Polarization microobjective 8 (focal distance $30 \mathrm{~mm}$, aperture 0.1 , magnification $4 \times$ ) was located at the focal length from the frequency plane of lens 7 and, thus, performed inverse Fourier transform of the filtered out polarization field of laser radiation. The coordinate distribution of the intensity of these fields, polarizationally filtered by the quarter-wave plate 9 and polarizer 10 , was registered in the plane of CCD-camera 11 (The Imaging Source DMK 41AU02.AS, monochrome 1/2" CCD, Sony ICX205AL (progressive scan); resolution 1280×960; light sensitive area size $7600 \times 6200 \mu \mathrm{m}$; sensitivity $0.05 \mathrm{~lx}$; dynamic range 8 bit; SNR 9 bit, deviation of photosensitive characteristics from linear is no more than 15\%). It provided the range of measuring the structural elements of the polycrystalline network with the resolution of $2 \ldots 2000 \mu \mathrm{m}$.
For the series of linearly $\left(0^{\circ} ; 45^{\circ} ; 90^{\circ}\right)$ and righthand $(\otimes)$ circularly polarized probing laser beams $\left(\lambda_{1}\right.$ and $\lambda_{2}$ ), the Stokes-vector parameters $S_{i=2 ; 3 ; 4}^{0 ; 45 ; 90 \otimes}$ were measured in the points of the digital image

$$
\left\{\begin{array}{l}
S_{i=2}^{0 ; 45 ; 90 ; \otimes}\left(\lambda_{1}, \lambda_{2}\right)=I_{0}{ }^{0 ; 45 ; 90 ; \otimes}+I_{90}{ }^{0 ; 45 ; 90 ; \otimes} ; \\
S_{i=2}^{0 ; 45 ; 90 ; \otimes}\left(\lambda_{1}, \lambda_{2}\right)=I_{0}{ }^{0 ; 45 ; 90 ; \otimes}-I_{90}{ }^{0 ; 45 ; 90 ; \otimes} ; \\
S_{i=3}^{0 ; 45 ; 90 ; \otimes}\left(\lambda_{1}, \lambda_{2}\right)=I_{45}^{0 ; 45 ; 90 ; \otimes}-I_{135}^{0 ; 45 ; 90 ; \otimes} ; \\
S_{i=4}^{0 ; 45 ; 90 ; \otimes}\left(\lambda_{1}, \lambda_{2}\right)=I_{\otimes}{ }^{0 ; 45 ; 90 ; \otimes}-I_{\oplus}{ }^{0 ; 45 ; 90 ; \otimes} .
\end{array}\right.
$$

Here, $I_{0 ; 90 ; 45 ; 135 ; \otimes ; \oplus}^{0 ; 45 ; 90 \otimes}$ are the intensities of linearly $\left(0^{\circ}, 45^{\circ}, 90^{\circ}, 135^{\circ}\right)$, right- $(\otimes)$ and left-hand $(\oplus)$ circularly polarized components of the filtered (by means of polarizer 10 and quarter-wave plate 9) laser radiation.

Further, the Mueller-matrix invariants were calculated (PC 10)

$$
\left\{\begin{array}{l}
M_{14}\left(\lambda_{2}\right)=S_{1}^{\otimes}-0.5\left(S_{1}^{0}+S_{1}^{90}\right), \\
M_{41}\left(\lambda_{2}\right)=0.5\left(S_{4}^{0}+S_{4}^{90}\right), \\
M_{44}\left(\lambda_{1}, \lambda_{2}\right)=S_{4}^{\otimes}-0.5\left(S_{4}^{0}+S_{4}^{90}\right), \\
\sum M_{22 ; 33}\left(\lambda_{1}\right)=M_{22}+M_{33}= \\
\quad=0.5\left(S_{2}^{0}-S_{2}^{90}\right)+S_{3}^{45}-0.5\left(S_{3}^{0}+S_{3}^{90}\right), \\
\Delta M_{23 ; 32}\left(\lambda_{1}\right)=M_{23}-M_{32}= \\
\quad=S_{2}^{45}-0.5\left(S_{2}^{0}+S_{2}^{90}\right)-0.5\left(S_{3}^{0}-S_{3}^{90}\right) .
\end{array}\right.
$$

The series of Figs. 2 to 5 present the results of the technique of Mueller-matrix mapping of polycrystalline liquor films, taken over 1 hour and 3 hours after death.

With a view to a possible use of the Mueller-matrix method in determining the time of death for each group of samples, the magnitude of the statistical moments $\bar{Z}_{i=1 ; 2 ; 3 ; 4}(q)$ and standard deviations $\pm \sigma \quad$ were determined by the average (within group 1 and group 2).

The following settings determine the accuracy of the time of death (ATD), the interval determines the accuracy of the method $(\Omega)$ and $\operatorname{ATD}(\Delta T)($ Table 1$)$.

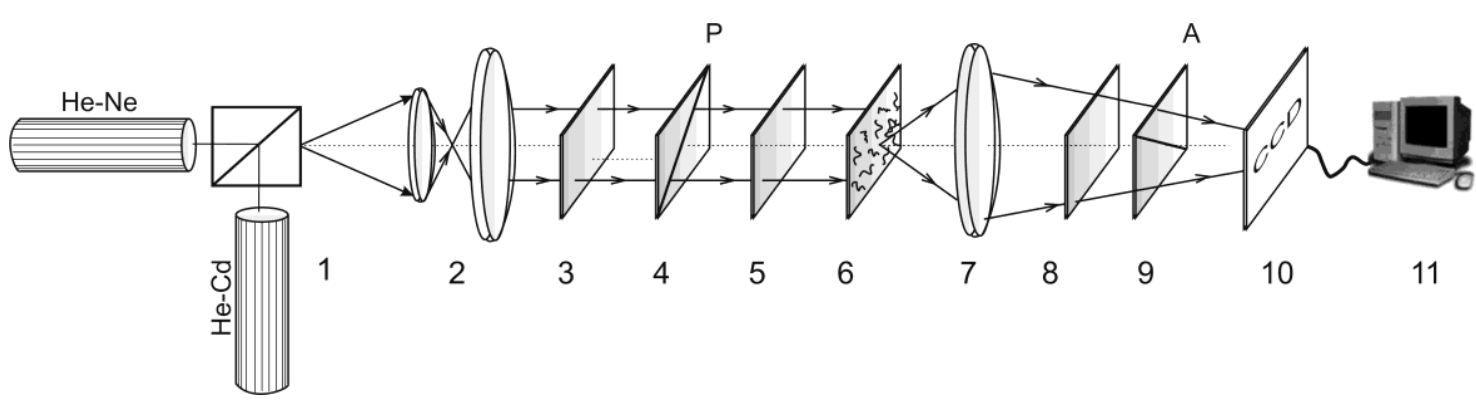

Fig. 1. Optical scheme of polarimeter, where $1-\mathrm{He}-\mathrm{Ne}(\mathrm{Cd})$ laser; 2 - collimator; 3 - stationary quarter-wave plate; $5,8-$ mechanically movable quarter-wave plates; 4, 9 - polarizer and analyzer, respectively; 6 - object of investigation; 7 polarization. 
Table 1. Timeframe and accuracy of the time of death.

\begin{tabular}{|c|c|c|}
\hline ATD, hours & $\Delta T$ & $\Omega$ \\
\hline 3 & 24 & \pm 0.1 \\
\hline 6 & 32 & \pm 0.15 \\
\hline 12 & 48 & \pm 0.2 \\
\hline
\end{tabular}

Table 2. Operational characteristics of the method of Mueller-matrix reconstruction.

\begin{tabular}{|c|c|c|c|c|c|}
\hline Parameters & $Z_{i}$ & Kidney & Liver & Pancreas & Spleen \\
\hline \multirow{5}{*}{$S e\left(Z_{i}\right), \%$} & $Z_{1}$ & $66.6 \%$ & $71.9 \%$ & $77.2 \%$ & $77.2 \%$ \\
\cline { 2 - 6 } & $Z_{2}$ & $70.2 \%$ & $70.2 \%$ & $78.9 \%$ & $82.4 \%$ \\
\cline { 2 - 6 } & $Z_{3}$ & $82.4 \%$ & $85.9 \%$ & $91.2 \%$ & $92.9 \%$ \\
\cline { 2 - 6 } & $Z_{4}$ & $80.7 \%$ & $89.4 \%$ & $94.7 \%$ & $94.7 \%$ \\
\hline \multirow{5}{*}{$\operatorname{Sp}\left(Z_{i}\right), \%$} & $Z_{1}$ & $63.1 \%$ & $66.6 \%$ & $73.6 \%$ & $73.6 \%$ \\
\cline { 2 - 6 } & $Z_{2}$ & $66.6 \%$ & $66.6 \%$ & $75.4 \%$ & $78.9 \%$ \\
\cline { 2 - 6 } & $Z_{3}$ & $80.7 \%$ & $82.4 \%$ & $87.8 \%$ & $89.4 \%$ \\
\cline { 2 - 6 } $\operatorname{Ac}\left(Z_{i}\right), \%$ & $Z_{4}$ & $77.2 \%$ & $85.9 \%$ & $91.2 \%$ & $92.9 \%$ \\
\hline \multirow{5}{*}{} & $Z_{1}$ & $64.85 \%$ & $69.25 \%$ & $75.4 \%$ & $75.4 \%$ \\
\cline { 2 - 6 } & $Z_{2}$ & $68.4 \%$ & $68.4 \%$ & $77.15 \%$ & $80.65 \%$ \\
\cline { 2 - 6 } & $Z_{3}$ & $81.55 \%$ & $84.15 \%$ & $89.5 \%$ & $91.15 \%$ \\
\cline { 2 - 6 } & $Z_{4}$ & $78.95 \%$ & $87.65 \%$ & $92.95 \%$ & $93.8 \%$ \\
\hline
\end{tabular}

Note. $\operatorname{Se}\left(Z_{i}\right)$ - sensitivity, $\operatorname{Sp}\left(Z_{i}\right)$ - specificity, $\operatorname{Ac}\left(Z_{i}\right)$ diagnostic test accuracy, $Z_{i}-$ the statistical moments of the $1^{\text {st }}$ $4^{\text {th }}$ orders that characterize the distribution of Mueller-matrix elements.

Table 2 illustrates the potential of this method when dealing with another problem of Experimental Medicine - differentiation of histological sections of the internal organs of healthy and diabetic rats.

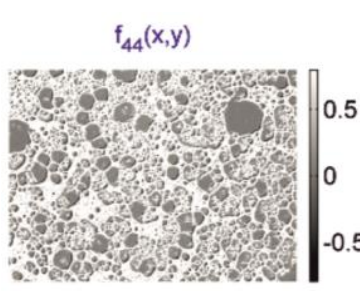

$\mathrm{f}_{44}(\mathrm{l}, \mathrm{x}, \mathrm{y})$

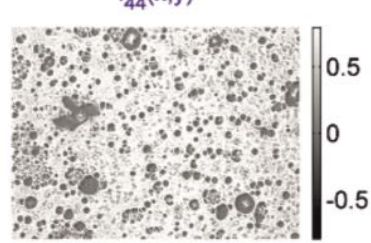

3)
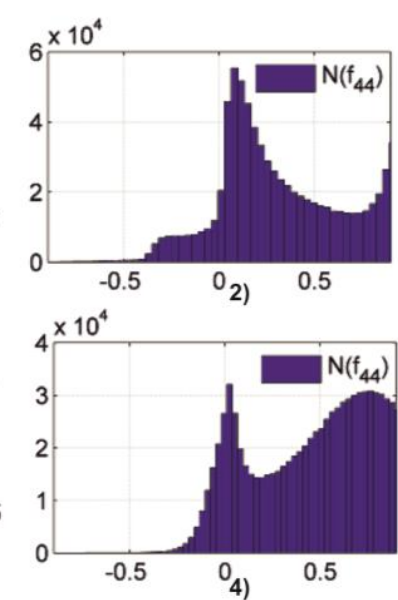

Fig. 2. Mueller-matrix image $f_{44}$ taken by the film of liquor 1 hour $(1,2)$ and 3 hours $(3$ and 4$)$ after death.
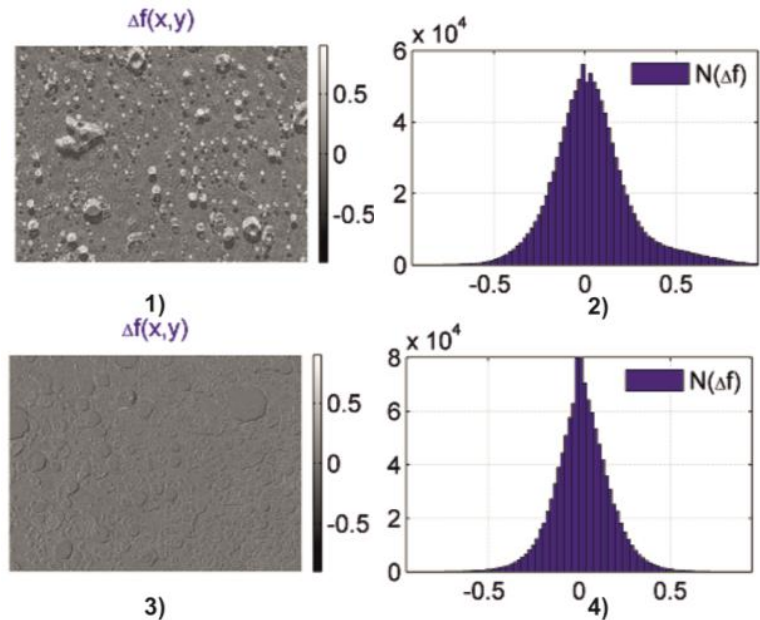

3)

Fig. 3. Mueller-matrix image $\Delta f$ taken by the film of liquor 1 hour $(1,2)$ and 3 hours $(3,4)$ after death.
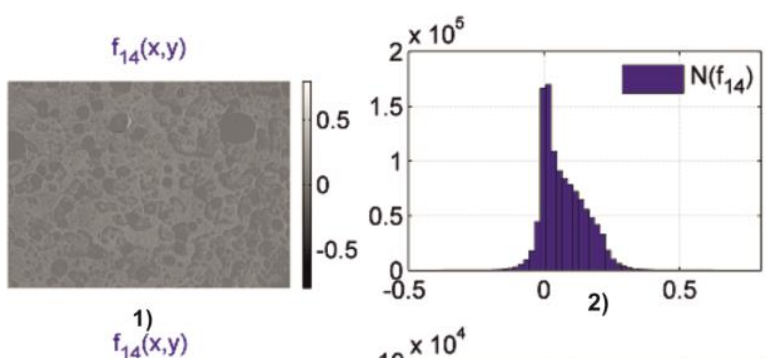

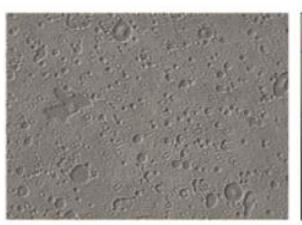

3)

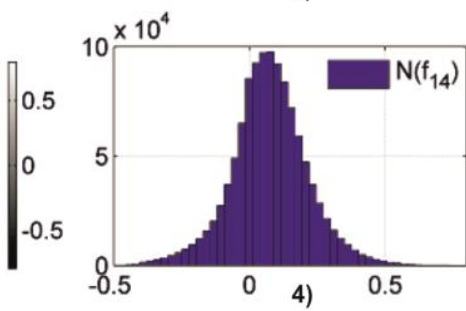

Fig. 4. Mueller-matrix image $f_{14}$ taken by the film of liquor 1 hour $(1,2)$ and 3 hours $(3,4)$ after death.

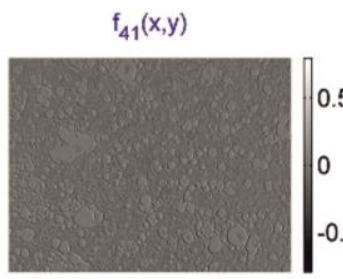

1)

$f_{41}(x, y)$

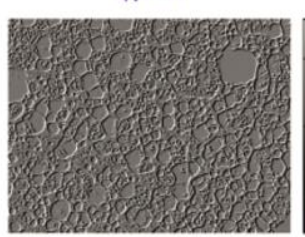

3)
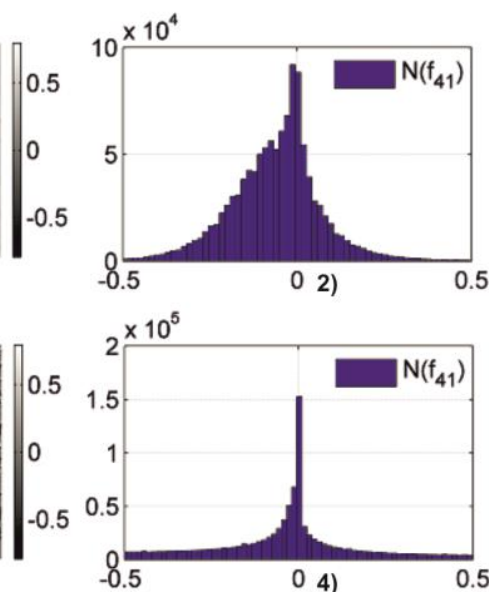

Fig. 5. Mueller-matrix image $f_{41}$ taken by the film of liquor in 1 hour $(1,2)$ and 3 hours $(3,4)$ after death. 


\section{Conclusions}

1. On the basis of the model of generalized optical anisotropy, the two-wave technique of azimuthally invariant Mueller-matrix reconstruction of optical anisotropy parameters that are characteristic of polycrystalline liquor smears has been developed.

2. The Mueller-matrix invariants characterizing polarization manifestations of different (partial) mechanisms of optical anisotropy of liquids smear have been determined.

3. The method for determining the time of death based on the time of monitoring changes in the optical anisotropy of the polycrystalline films of liquor has been proposed.

4. The interrelations between the set of statistical moments of the $1^{\text {st }}-4^{\text {th }}$ orders characterizing the distributions of optical anisotropy parameters and the difference in the structure of histological tissue sections of internal organs of rats have been determined.

\section{Acknowledgement}

This work was supported by the grants № 0113 U003239 and № 0112U002336 from the Ukrainian Foundation for Basic Researches.

\section{References}

1. M.H. Smith, P. Burke, A. Lompado, E. Tanner, L.W. Hillman, Mueller matrix imaging polarimetry in dermatology // Proc. SPIE, 3991, p. 210-216 (2000).

2. M.H. Smith, Interpreting Mueller matrix images of tissues // Proc. SPIE, 4257, p. 82-89 (2001).

3. Yu.A. Ushenko, A.P. Peresunko, and Bozan Adel Baku, A new method of Mueller-matrix diagnostics and differentiation of early oncological changes of the skin derma // Adv. Opt. Technol. 2010, 952423 (2010).

4. T.T. Tower, R.T. Tranquillo, Alignment maps of tissues: I. Microscopic elliptical polarimetry // Biophys. J. 81, p. 2954-2963 (2001).

5. J.M. Bueno, J. Jaronski, Spatially resolved polarization properties for in vitro corneas // Ophthal. Physiol. Opt. 21, p. 384-392 (2001).

6. J.M. Bueno, F. Vargas-Martin, Measurements of the corneal birefringence with a liquid-crystal imaging polariscope // Appl. Opt. 41, p. 116-124 (2002).

7. J.M. Bueno, M.C.W. Campbell, Polarization properties of the in vitro old human crystalline lens // Ophthal. Physiol. Opt. 23, p. 109-118 (2003).

8. T.T. Tower, R.T. Tranquillo, Alignment maps of tissues: II. Fast harmonic analysis for imaging // Biophys. J. 81, p. 2964-2971 (2001).

9. M. Shribak and R. Oldenbourg, Techniques for fast and sensitive measurements of two-dimensional birefringence distributions // Appl. Opt. 42, p. 3009-3017 (2003).
10. S.N. Savenkov, V.V. Marienko, E.A. Oberemok, O.I. Sydoruk, Generalized matrix equivalence theorem for polarization theory // Phys. Rev. E, 74, p. 605-607 (2006).

11. A.G. Ushenko and V.P. Pishak, Laser polarimetry of biological tissue: Principles and applications, in: Handbook of Coherent-Domain Optical Methods: Biomedical Diagnostics, Environmental and Material Science, 1, p. 93-138, ed. by V.V. Tuchin. Kluwer Academic Publishers, 2004.

12. Yu.A. Ushenko, Yu.Ya. Tomka, A.V. Dubolazov, Complex degree of mutual anisotropy of extracellular matrix of biological tissues // Optika $i$ Spektroskopiya, 110, p. 814-819 (2011), in Russian.

13. Yu.A. Ushenko, Investigation of formation and interrelations of polarization singular structure and Mueller-matrix images of biological tissues and diagnostics of their cancer changes // J. Biomed. Opt. 16, 066006 (2011).

14. Yu.A. Ushenko, I.Z. Misevich, O.Yu. Telenha, Yu.Ya. Tomka, A.O. Karachevtsev, Polarizationsingular structure of laser images of stratified phase-inhomogeneous layers for the diagnostics and classification of their optical properties // Optical Memory and Neural Networks (Information Optics), 20, p. 59-70 (2011).

15. Yu.A. Ushenko, Yu.Ya. Tomka, A.V. Dubolazov, O.Yu. Telenha, Diagnostics of optical anisotropy changes in biological tissues using Müller matrix // Quantum Electronics, 41(3), p. 273-277 (2011).

16. Yu.A. Ushenko, A.V. Dubolazov, A.O. Karachevtsev, Statistical structure of skin derma Mueller matrix images in the process of cancer changes // Optical Memory and Neural Networks (Information Optics), 20, p. 145-154 (2011).

17. O.V. Angelsky, Yu.A. Ushenko, A.V. Dubolazov, O.Yu. Telenha, The interconnection between the coordinate distribution of Mueller-matrixes images characteristic values of biological liquid crystals net and the pathological changes of human tissues // Adv. Opt. Technol. 2010, Article ID 130659, 10 pages (2010).

18. Yu.A. Ushenko, Yu.Ya. Tomka, A.V. Dubolazov, V.A. Balanetskaya, V.P. Unguryan, N.I. Zabolotna, B.P. Oleinichenko, Mueller-matrix diagnostics of optical properties inherent to polycrystalline networks of human blood plasma // Semiconductor Physics, Quantum Electronics \& Optoelectronics, 14(1), p. 98-105 (2011).

19. Yu.A. Ushenko, A.V. Dubolazov, V.A. Balanetskaya, A.O. Karachevtsev, V.A. Ushenko, Wavelet-analysis of polarization maps of human blood plasma // Optika $i$ Spektroskopiya, 113(3), p. 332-343 (2012), in Russian.

20. O.V. Angelsky, P.V. Polyanskii, C.V. Felde, The emerging field of correlation optics // Optics and Photonics News, 23(4), p. 25-29 (2012). 
21. O.V. Angelsky, A.Ya. Bekshaev, P.P. Maksimyak, A.P. Maksimyak, S.G. Hanson, C.Yu. Zenkova, Self-diffraction of continuous laser radiation in a disperse medium with absorbing particles // Opt. Exp. 21(7), p. 8922-8938 (2013).

22. O.V. Angelsky, G.V. Demianovsky, A.G. Ushenko, D.N. Burkovets, Y.A. Ushenko, Wavelet analysis of two-dimensional birefringence images of architectonics in biotissues for diagnosing pathological changes // J. Biomed. Opt. 9(4), p. 679-690 (2004).

23. Angel'skii, A.G. Ushenko, S.B. Ermolenko, D.N. Burkovets, Yu.A. Ushenko, O.V. Pishak, Polarization-based visualization of multifractal structures for the diagnostics of pathological changes in biological tissues // Optika i Spektroskopiya, 89(5), p. 799-804 (2000), in Russian.

24. Yu.A. Ushenko, Yu.Ya. Tomka, A.V. Dubolazov, Laser diagnostics of anisotropy in birefringent networks of biological tissues in different physiological conditions // Quantum Electronics, 41(2), p. 170-175 (2011).

25. Yu.O. Ushenko, Yu.Ya. Tomka, O.V. Dubolazov, V.O. Balanets'ka, A.V. Karachevtsev, A.P. Angelsky, Wavelet-analysis for laser images of blood plasma // AECE - Adv. in Electr. and Comput. Eng. 11(2), (2011).

26. V.T. Bachinsky, Yu.O. Ushenko, Yu.Ya. Tomka, O.V. Dubolazov, V.O. Balanets'ka, A.V. Karachevtsev, Wavelet analysis for polarization maps of networks formed by liquid biological crystals in blood plasma: statistical and fractal approaches // Semiconductor Physics, Quantum Electronics \& Optoelectronics, 13(2), p. 189-201 (2010).

27. Yu.O. Ushenko, O.V. Dubolazov, A.O. Karachevtsev, M.P. Gorsky, Yu.F. Marchuk, Wavelet analysis of Fourier polarized images of the human bile // Appl. Opt. 51, p. C133-C139 (2012). 